\title{
Short Homophobia Scale (SHS): desempenho em estudantes universitárias de enfermagem
}

Homophobia Short Scale (HSS): Performance in female nursing students Short Homophobia Scale (SHS): desempeño en estudiantes universitarias de enfermeria

\author{
Zuleima Cogollo-Milanés ${ }^{1}$ id https://orcid.org/0000-0003-3310-4052 \\ Adalberto Campo-Arias² io https://orcid.org/0000-0003-2201-7404 \\ Edwin Herazo ${ }^{3}$ io https://orcid.org/0000-0002-9461-7997
}

Como citar:

Cogollo-Milanés Z, Campo-Arias A, Herazo E. Short Homophobia Scale (SHS): desempenho em estudantes universitárias de enfermagem. Acta Paul Enferm. 2021;34:eAPE02692.

DOI http://dx.doi.org/10.37689/actaape/2021A002692

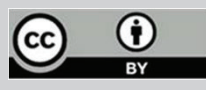

Descritores

obia; Estudos

de validação

Keywords

Students nursing; Homophobia; Validation studies;

Descriptores

Estudiantes de enfermería; Homofobia; Estudio de

validación

Submetido 13 de setembro de 2019 Aceito

20 de agosto de 2020

\section{Autor correspondente}

Zuleima Cogollo

E-mail: zcogollom@ucartagena.edu.co

\section{Resumo}

Objetivo: Estimar o desempenho psicométrico da escala de homofobia Short Homophobia Scale (SHS) em estudantes de enfermagem do sexo feminino de uma universidade em Cartagena, Colômbia.

Métodos: Desenhou-se um estudo de validação que incluiu 419 estudantes de enfermagem do sexo feminino cursando do primeiro ao oitavo semestre com 18 a 29 anos de idade $(M=20,9 ; D P=2,9)$. As estudantes preencheram os quatro itens da SHS. Observou-se que 0 instrumento tem consistência interna (alfa de Cronbach e ômega de McDonald) e dimensionalidade (análise fatorial confirmatória, AFC).

Resultados: Considerou-se a consistência interna aceitável (Cronbach 0,68 e McDonald 0,69). Na AFC, um fator retido explicou $51,5 \%$ da variância. Os indicadores globais de qualidade do ajuste foram aceitáveis (raiz quadrada da média do erro de aproximação $=0,08$; IC $=90 \%$ 0,03 a 0,14; índice de ajuste comparativo $=0,98$; índice de Tucker-Lewis $=0,94$; e raiz quadrada média residual padronizada $=0,02$ ). Os escores foram semelhantes por etnia/raça e orientação política $(p>0,001)$ e significativamente mais elevados em estudantes cristãs que em outras afiliações religiosas $(p<0,001)$.

Conclusão: Em estudantes de enfermagem do sexo feminino, o desempenho da escala SHS foi considerado aceitável pela consistência interna e pela observância de um fator, havendo também bons indicadores de dimensionalidade. É necessário pesquisar o desempenho em alunos do sexo masculino.

\section{Abstract}

Objective: To estimate the psychometric performance of the Homophobia Short Scale (HSS) in female nursing students in a university in Cartagena, Colombia.

Method: A validation study was designed in which 419 female nursing students from the first to the eighth semester participated, aged between 18 and 29 years $(M=20.9, S D=2.9)$. The students completed all four items of the HSS. Internal consistency was found (Cronbach's alpha and McDonald's omega) and dimensionality (confirmatory factor analysis, CFA).

Results: The internal consistency was acceptable (Cronbach of 0.68 and McDonald of 0.69) and in the CFA one factor was retained which accounted for $51.5 \%$ of the variance, with acceptable global indicators of goodness of fit (the root of the mean square error of approximation $=0.08, \mathrm{Cl}=90 \% 0.03-0.14$, Comparative Fit Index $=0.98$, Tucker-Lewis Index $=0.94$, and standardized mean square residual $=0.02$ ). The scores were similar, according to ethnic-race and political orientation ( $p>0.001$ ) and significantly higher in Christians than in other religious affiliations $(p<0.001)$. 
Conclusion: In female nursing students, the HSS shows an acceptable performance in internal consistency and one factor, with good dimensionality indicators. It is necessary to know this performance in male nursing students.

\section{Resumen}

Objetivo: Estimar el desempeño psicométrico de la escala de homofobia Short Homophobia Scale (SHS) en estudiantes de enfermería de sexo femenino de una universidad en Cartagena, Colombia.

Métodos: Estudio de validación que incluyó 419 estudiantes de enfermería de sexo femenino, cursando desde el primer al octavo semestre, de 18 a 29 años de edad $(M=20,9 ; D P=2,9)$. Las estudiantes completaron los cuatro ítems de la SHS. Se observó que el instrumento tiene consistencia interna (alfa de Cronbach y omega de McDonald) y dimensionalidad (análisis factorial confirmatorio, AFC).

Resultados: La consistencia interna fue considerada aceptable (Cronbach 0,68 y McDonald 0,69). En el AFC, un factor retenido fue el motivo del 51,5 \% de varianza. Los indicadores globales de calidad del ajuste fueron aceptables (raíz cuadrada del promedio del error de aproximación = 0,08; IC = 90 \% 0,03 a 0,14; índice de ajuste comparativo =0,98; índice de Tucker-Lewis =0,94; y raíz cuadrada promedio residual estándar =0,02). La puntuación fue semejante por etnia/raza y orientación política ( $p>0,001)$ y significativamente más elevada en estudiantes cristianas que en otras religiones ( $p>0,001)$.

Conclusión: El desempeño de la escala SHS en estudiantes de enfermería de sexo femenino fue considerado aceptable por la consistencia interna y por el cumplimiento de un factor. También se observaron buenos indicadores de dimensionalidad. Es necesario estudiar el desempeño en alumnos de sexo masculino.

\section{Introdução}

O termo "homofobia" se refere à atitude negativa de aversão, condenação, rejeição ou proscrição contra pessoas homossexuais. ${ }^{(1)}$ Porém, como a homofobia não é clinicamente uma fobia; e sim, um preconceito contra a orientação sexual, ${ }^{(2)}$ propóe-se outras denominações, como "homonegatividade"(3) ou "homopreconceito". (4) Assim como outros termos, porém, o termo "homofobia" foi e continua sendo usado na literatura científica e popular em detrimento de sua raiz etimológica. ${ }^{(5)}$

Atualmente existem várias escalas que quantificam a atitude em relação a pessoas homossexuais: Index of Homophobia ou Attitudes Toward Homosexuality Scale, ${ }^{(3)}$ Homophobia Scale (HS-7), ${ }^{(6)}$ Attitudes Toward Lesbians and Gay Men Scale, ${ }^{(7)}$ Prejudicial Evaluation Scale, ${ }^{(8)}$ Modern Homophobia Scale, ${ }^{(9)}$ Homonegativity Scale, ${ }^{(10)}$ Homophobia Scale ${ }^{(11)}$ e Modern Homonegativity Scale. ${ }^{(12)}$ A HS$7^{(6)}$ e a Escala Multidimensional de Atitudes face a Lésbicas e a Gays ${ }^{(7)}$ são os instrumentos mais usados em pesquisa, apesar de suas limitaçóes. ${ }^{(13,14)}$

A HS-7 é um instrumento breve desenvolvido por Bouton et al. cujo propósito é quantificar a atitude em relação a pessoas homossexuais. ${ }^{(6)}$ A HS-7 já foi muito usada em diferentes pesquisas com alunos universitários no contexto global. ${ }^{(13-15)} \mathrm{Na}$ Colômbia, o desempenho da HS-7 foi avaliado em alunos de medicina em cidades como Bogotá e Bucaramanga. ${ }^{(15-17)}$ Uma versão em espanhol foi usada, cujo processo de tradução, traduçãao reversa e ajuste para uso em espanhol na Colômbia foi relatado em outra publicação. ${ }^{(18)} \mathrm{Na}$ escala HS-7, explora-se a atitude frente à homossexualidade com afirmaçóes (tradução livre para o português de trechos): "a homossexualidade é nojenta", "os homossexuais tem a mesma moralidade dos heterossexuais", "os homossexuais corrompem os jovens", "os homossexuais deveriam ter igualdade de direitos civis", "a homossexualidade é um pecado", "os homossexuais contribuem positivamente para a sociedade" e "a homossexualidade deveria ser ilegal". Cada item tem cinco opçóes de resposta que valem de um a cinco pontos e vão de "discordo plenamente" a "concordo plenamente" - quanto maior o escore, mais negativa é a atitude em relação à homossexualidade. ${ }^{(19)}$

Assim como outras escalas que medem esse construto, esse instrumento, porém, apresentou um desempenho abaixo do padrão esperado, com soluçôes fatoriais abaixo de $50 \%$ e sem análise fatorial confirmatória (AFC). ${ }^{(15-17)}$ Começando, portanto, pela observação da AFC, Campo-Arias et al. iniciaram um processo de aperfeiçoamento e propuseram uma versão com apenas quatro itens, a SHS; a nova escala SHS apresentou uma consistência interna elevada (alfa de Cronbach e ômega de McDonald de 0,77 ) e uma solução fatorial melhor, com apenas um fator que explicou 59,7\% da variância total. ${ }^{(19)}$

Nesse estudo, testou-se o desempenho da SHS em estudantes de enfermagem do sexo feminino, tem em mente não apenas um melhor desempenho psicométrico, mas também a vantagem que é conferida pela brevidade das escalas em processos de pesquisa. É mais confortável aplicar, qualificar e interpretar os instrumentos; demanda-se menos tempo para preencher; e os entrevistados ficam menos 
fatigados - o que ajuda a garantir indicadores de confiabilidade e validade melhores na medição. ${ }^{(20)}$

No tocante ao desempenho nomológico da SHS, empregou-se neste estudo a análise de variância (ANOVA, teste F) para comparar as médias e os desvios-padrão a afiliações religiosas; assumiu-se que cristãs não católicas teriam escores maiores que as católicas ou que as praticantes de outras religióes.

A homofobia está presente em todas as áreas da vida diária, inclusive em estudantes universitários que, em decorrência de sua escolaridade, deveriam ter uma atitude positiva em relação à diversidade sexual, especialmente os das ciências da saúde. ${ }^{(21,22)} \mathrm{A}$ homofobia, como outros preconceitos, é um estressor diário na vida das pessoas homossexuais ${ }^{(23)}$ e tem impactos negativos nas pessoas, nas comunidades e na sociedade em geral, visto que afeta negativamente a saúde mental e explica muitas das desigualdades em saúde vividas pelas pessoas gays. ${ }^{(24)}$

No tocante a estudantes de enfermagem - e se considerando as características dessa profissão, que oferece um apoio integrativo e holístico das dimensôes biológica, psicológica, social, cultural e ética - e no tocante às concepçóes, percepçóes e valores da pessoa que é o ponto inicial da assistência, deve haver uma atitude favorável à comunidade LGBT. Portanto, atitudes negativas limitam a possibilidade de assistência individualizada e integral: a homofobia é uma barreira na relação entre a equipe de enfermagem e pacientes gays, lésbicas, bissexuais e transgêneros. ${ }^{(25)}$ Portanto, medir atitudes homofóbicas nos estudantes de enfermagem é muito importante, dada a elevada incidência de preconceito sexual nesse grupo de profissionais. ${ }^{(26)}$ Estudos que oferecem informaçóes sobre a magnitude da situação possibilitam às instituiçóes educativas a adoção de medidas precisas e tempestivas para reduzir o impacto negativo dos preconceitos sexuais nessa prática. ${ }^{(27)}$

Ainda não se determinou o desempenho psicométrico da SHS em estudantes de enfermagem e, por isso, é necessário ter informaçôes que garantam a confiabilidade e a validade nessa área do ensino superior.

Esta pesquisa visa estimar o desempenho psicométrico, a consistência interna e a dimensionalidade da SHS em estudantes de enfermagem de uma universidade em Cartagena, Colômbia.

\section{Métodos}

\section{Desenho}

Realizou-se um estudo metodológico a partir de uma pesquisa de triagem com um instrumento respondido a lápis e papel. $\mathrm{O}$ estudo foi aprovado pelo comitê de ética da universidade. Dadas as características do estudo, ele se enquadra como investigação livre de riscos na Resolução n.o 8430 de 1993 do Ministério da Saúde da Colômbia. ${ }^{(28)}$

\section{Amostra}

Neste estudo, solicitou-se a participação de estudantes de enfermagem de uma universidade pública em Cartagena, Colômbia. A pesquisa contou com a colaboração voluntária de 419 estudantes do sexo feminino cursando do primeiro ao oitavo semestre. Esse número de participantes foi ideal para se aplicar a AFC e calcular os coeficientes habituais para se estabelecer a consistência interna. ${ }^{(29,30)}$ As participantes tinham entre 18 e 29 anos de idade $(M=20,9 ; D P=2,9)$. A distribuição dos semestres em que as estudantes estavam foi a seguinte: primeiro, 71 estudantes (16,9\%); segundo, 82 (19,6\%); terceiro, 50 (11,9\%); quarto, 54 (12,9\%); quinto, 79 (18,9\%); sexto, 44 (10,5\%); sétimo, 18 (4,3\%); e oitavo, 21 (5,0\%). Quanto ao estado civil, 376 estudantes (89,7\%) relataram ser solteiras; e 43 (10,3\%), casadas ou vivendo em uniāo livre. A distribuição etnicorracial autodeclarada foi de 271 estudantes mestiças (64,7\%); 89 afrocolombianas $(21,2 \%) ; 21$ indígenas $(5,0 \%)$; 38 não declaradas $(9,1 \%)$. No tocante à religiáo, 300 estudantes (71,6\%) relataram ser adeptas do catolicismo; 103 (24,6\%) de outras correntes cristãs; e 16 (3,6\%) declararam não ter religião. No tocante à orientação política, a distribuição foi a seguinte: 181 estudantes liberais (43,2\%), 123 conservadoras $(29,4 \%)$; 37 socialistas $(8,8 \%)$; e 78 declararam não ter nenhuma orientação política $(18,6 \%)$.

\section{Instrumentos}

O questionário da pesquisa abarcava as informaçóes demográficas acima e as perguntas da SHS. O instrumento de medição é composto por quatro itens: "a homossexualidade é nojenta", "os homossexuais corrompem os jovens", "a homossexualidade é um 
pecado" e "a homossexualidade deveria ser ilegal". Nele, constam cinco opçóes de resposta que vão de "discordo plenamente" a "concordo plenamente". As respostas vão de um a cinco pontos, de modo que o escore total fique entre quatro e vinte. Um escore mais baixo sugere uma atitude mais positiva em relação à homossexualidade. ${ }^{(19)}$

\section{Processo}

As estudantes preencheram o questionário da pesquisa em sala de aula, em um formulário. O questionário foi entregue em um envelope em branco. Orientou-se que as estudantes omitissem nomes e quaisquer informaçóes que possibilitassem uma identificação. Uma equipe de assistentes de pesquisa explicou os objetivos do estudo, como responder o questionário e sanou as dúvidas sobre os itens da SHS, sem condicionar as respostas das participantes. Os assistentes também informaram as estudantes de que poderiam não participar — não receber o envelope, não responder pontos que as deixassem desconfortáveis ou devolver o formulário não respondido, se assim desejassem —, sem que isso implicasse qualquer tratamento negativo por parte dos pesquisadores. Terminado o questionário, as estudantes deveriam colocá-lo de volta no envelope e devolvê-lo aos assistentes. A confiabilidade foi preservada no processo de reunião das informaçóes, pois os questionários eram anônimos. Essas informaçôes foram coletadas entre $1 .^{\circ}$ de março e 31 de maio de 2018.

\section{Análise estatística}

Aplicou-se uma análise AFC para corroborar a estrutura unidimensional da SHS. Esse processo foi feito pelo método máxima verossimilhança. Aplicou-se também o teste de Kaiser-Meyer-Olkin (KMO) para se confirmar a adequacidade da $\operatorname{amostra}^{(31)}$ e o teste de esfericidade de Bartlett. ${ }^{(32)}$ Concluiu-se a análise AFC com o cálculo do coeficiente RMSEA, ou raiz quadrada da média do erro de aproximação (intervalo de confiança de 90\% [IC = 90\%]), do índice de ajuste comparativo (CFI), do índice de Tucker-Lewis (TLI) e da raiz quadrada média residual padronizada (SRMR). A RMSEA e a SRMR XYZ devem ser abaixo de 0,06; e o $C F I$ e o TLI
XYZ devem ser acima de 0,89. Calculou-se o alfa de Cronbach $^{(33)}$ e o ômega de McDonald ${ }^{(34)}$ para se quantificar a consistência interna. Comparou-se também os escores da SHS à declaração etnicorracial e às afiliaçôes religiosa e política pela análise de variância (ANOVA, teste F). Como esse teste é muito sensível ao tamanho da amostra, os valores de $\mathrm{p}$ inferiores a $0,001^{(35)}$ foram aceitos como significativos. A análise foi feita no programa estatístico STATA 13.0. ${ }^{(36)}$

\section{Resultados}

Os escores da SHS ficaram entre 4 e $20(M=10,1 ; D P$ $=3,7)$. Os coeficientes de consistência interna foram: alfa de Cronbach de 0,68 e ômega de McDonald de 0,69 . Vide mais informações na tabela 1 .

Tabela 1. Média, desvio-padrão (DP), correlação item/total corrigida (CIIC) e alfa de Cronbach se excluído (CAID) por item da SHS

\begin{tabular}{llll}
\hline Item & Média (DP) & CIIC & CAID \\
\hline 1 & $2,31(1,24)$ & 0,48 & 0,60 \\
2 & $2,50(1,20)$ & 0,52 & 0,58 \\
3 & $3,05(1,49)$ & 0,42 & 0,65 \\
4 & $2,22(1,18)$ & 0,45 & 0,62 \\
\hline
\end{tabular}

$\mathrm{Na}$ análise AFC, o teste de esfericidade de Bartlett apresentou qui-quadrado igual a 254,8 com 6 graus de liberdade; a probabilidade foi inferior a 0,001 . Identificou-se apenas um fator com eigenvalue igual a 2,1 que correspondeu a $51,5 \%$ da variância. $\mathrm{O}$ indicador da qualidade do ajuste foi RMSEA $=0,08(\mathrm{IC}=90 \%$; 0,03 a 0,14); CFI =0,98; e $T L I=$ 0,94 . Na tabela 2, estão dispostas as comunalidades e cargas desses fatores.

Tabela 2. Comunalidade e cargas dos itens da SHS

\begin{tabular}{lcc}
\hline Item & Comunalidade & Carga \\
\hline 1 & 0,399 & 0,632 \\
2 & 0,442 & 0,655 \\
3 & 0,272 & 0,522 \\
4 & 0,311 & 0,558 \\
\hline
\end{tabular}

Esses escores estão dispostos na tabela 3 por declaração etnicorracial, afiliação religiosa e orientação política. As estudantes afiliadas aos grupos não católicos apresentaram escores significativamente maiores que as da crença católica ou sem religiáo 
determinada. As diferenças por grupo etnicorracial ou orientação política não foram estatisticamente significativas.

Tabela 3. Comparação dos escores da SHS a algumas características e valores de F e p (ANOVA)

\begin{tabular}{lcccc}
\hline Variável & $\mathbf{M}$ & $\mathbf{D P}$ & $\mathbf{F}$ & Valor de $\mathbf{p}^{\text {* }}$ \\
\hline Etnia/raça & & & & \\
$\quad$ Outros & 10,7 & 4,8 & 2,36 & 0,095 \\
$\quad$ Afrocolombiana & 10,6 & 3,6 & & \\
$\quad$ Mestiça & 9,8 & 3,6 & & \\
Afiliação religiosa & & & & \multirow{2}{*}{$0,001^{*}$} \\
$\quad$ Cristã & 12,3 & 3,9 & 29,3 & \\
$\quad$ Católica & 9,4 & 3,2 & & \\
$\quad$ Ausente & 8,2 & 3,9 & & \\
Orientação política & & & & \\
$\quad$ Ausente & 10,5 & 3,6 & 2,75 & \\
$\quad$ Conservadora & 10,4 & 3,8 & & \\
$\quad$ Liberal & 9,9 & 3,4 & & \\
$\quad$ Socialista & 8,6 & 4,2 & & \\
\hline
\end{tabular}

*Diferença estatisticamente significativa.

\section{Discussão}

Neste estudo, observa-se que a SHS apresentou uma consistência interna e indicadores da qualidade do ajuste aceitáveis no conjunto de aspectos que abarcou as estudantes de enfermagem da cidade de Cartagena, Colômbia.

Constatou-se a consistência interna da SHS pelos valores do alfa de Cronbach e do ômega de McDonald, respectivamente 0,68 e 0,69; esses achados são inferiores aos observados no único outro estudo que avaliou o desempenho da SHS; no estudo de Campo-Arias et al., o valor relatado do alfa de Cronbach e do ômega de McDonald foi $0,77 . .^{(20)} \mathrm{Em}$ condiçóes ideais, espera-se que os valores de consistência interna fiquem entre 0,70 e 0,95. ${ }^{(29,37)}$ Esses achados, porém, devem ser interpretados sob a consideração de que podem variar dependendo da população; assim, torna-se necessário validá-los dentro de cada grupo e se considerando suas características particulares, como no caso das estudantes de enfermagem. ${ }^{(38)}$

$\mathrm{O}$ estudo corroborou a dimensionalidade $\mathrm{da}$ SHS, e a solução fatorial apresentou um único fator que explicou 51,5\% da variância total. Em Campo et al., observou-se uma porcentagem ligeiramente superior de variância: a solução fatorial da SHS explicou $59,7 \%$ da variância total. ${ }^{(20)}$ Esse achado corrobora a unidimensionalidade, dado que a variância explicada é superior a 50\%. ${ }^{(39)}$ A heterogeneidade das populaçóes pode explicar a diferença de variância nos estudos, dadas as diferentes cidades do país e diferentes características culturais. ${ }^{(40)}$

No tocante ao desempenho nomológico, observou-se uma significância estatística entre os escores homofóbicos e ter uma religião cristã não católica, o que não se observou nas estudantes de outras afiliações religiosas. Esse achado condiz com outros estudos que relataram que estudantes praticantes de alguma religiáo ou que participam de cultos religiosos apresentavam escores de homofobia maiores que os menos envolvidos religiosamente. ${ }^{(41,42)}$ As religióes cristãs condenam a homossexualidade e a consideram um pecado abominável. ${ }^{(43)}$

Este é o primeiro estudo deste tipo que comprova o desempenho psicométrico da SHS em estudantes de enfermagem, portanto, é uma contribuição essencial à quantificação da homofobia, um aspecto relevante nesse grupo de futuros profissionais da saúde se considerarmos que, na assistência prestada por esses profissionais, os indivíduos com orientação sexual diferente da heterossexual poderão ser vítimas de estigma, preconceito e discriminação. ${ }^{(44)}$ Uma limitação, porém, é que o estudo incluiu apenas mulheres (dada a pequena quantidade de homens que estudam enfermagem na universidade em que o estudo foi conduzido), sendo que já se observou uma diferença nas atitudes de homens e mulheres em relação a orientaçóes sexuais não hegemônicas - em geral, as mulheres têm uma atitude mais positiva em relação à homossexualidade. ${ }^{(1)}$

Nesse sentido, deduzimos a importância deste estudo no âmbito acadêmico e de pesquisa. É necessário ter uma escala breve como a SHS, capaz de apresentar uma quantificação válida e confiável da homofobia em pesquisas futuras e também coletar informaçóes exatas sobre a homofobia, não apenas no campo da educação de enfermeiros, como também entre os que já praticam a profissão, ${ }^{(45)}$ pois essa forma de rejeição constitui uma barreira à prestação de uma assistência humanizada e digna para o paciente. A homofobia é um estressor na vida diária dos homossexuais. ${ }^{(23)}$ Como outros estressores, a homofobia aumenta o risco de problemas emocionais em pessoas que a enfrentam diariamente. ${ }^{(46,47)}$ 
As atitudes de rejeição dessa população afetam, dentro do escopo da profissão, os usuários dos serviços de saúde e também qualificam a interação com outras pessoas nos diferentes contextos em que os estudantes ou profissionais possam desenvolver a prática, como colegas de estudo ou trabalho, vizinhos ou pessoas comuns que possam ter orientaçóes homossexuais. ${ }^{(27)}$

\section{Conclusão}

Conclui-se que a SHS é um instrumento com consistência interna elevada. Mesmo assim, não se pôde comprovar a dimensionalidade com todos os indicadores. É necessário revisar a validade do construto da SHS em outros contextos e revisar o desempenho da SHS em alunos de enfermagem do sexo masculino.

\section{Agradecimentos}

Agradecemos às estudantes de enfermagem que participaram do estudo na Universidad de Cartagena (Z. Cogollo), Universidad del Magdalena (Adalberto Campo-Arias) and Instituto de Investigación del Comportamiento Humano (Edwin Herazo).

\section{Colaborações}

Cogollo Z, Campo A e Herazo E contribuíram com a concepção do estudo, análise e interpretação dos dados, redação do artigo, revisão crítica relevante do conteúdo intelectual e aprovação da versão final a ser publicada.

\section{Referências}

1. Fone B. Homofobia. Una historia. México: Océano; 2008.

2. Herek GM. The psychology of sexual prejudice. Curr Dir Psychol Sci. 2000;9(1):19-22.

3. Hudson WW, Ricketts WA. A strategy for the measurement of homophobia. J Homosex. 1980;5(4):357-72.

4. Logan CR. Homophobia? No, homoprejudice. J Homosex. 1996;31(3):31-53.
5. Isay R. Being homosexual: Gay men and their development. New York: Avon Books; 1989.

6. Bouton RA, Gallaher PE, Garlinghouse PA, Leal T, Rosenstein LD, Young RK. Scales for measuring fear of AIDS and homophobia. J Pers Assess. 1987;51(4):606-14.

7. Herek GM. Heterosexuals' attitudes toward lesbians and gay men: correlates and gender differences. J Sex Res. 1988;25(4):45177.

8. Kelly JA, St Lawrence JS, Smith S Jr, Hood HV, Cook DJ. Stigmatization of AIDS patients by physicians. Am J Public Health. 1987l;77(7):78991.

9. Raja S, Stokes JP. Assessing attitudes toward lesbians and gay men: The Modern Homophobia Scale. J Gay Lesbian Bisex Identity. 1998;3:113-34.

10. Morrison TG, Parriag AV, Morrison MA. The psychometric properties of the Homonegativity Scale. J Homosex. 1999;37(4):111-26.

11. Wright LW Jr, Adams HE, Bernat J. Development and validation of the Homophobia Scale. J Psychopathol Behav Assess. 1999;21(4):33747.

12. Morrison MA, Morrison TG. Development and validation of a scale measuring modern prejudice toward gay men and lesbian women. J Homosex. 2002;43(2):15-37.

13. Grey JA, Robinson BB, Coleman E, Bockting WO. A systematic review of instruments that measure attitudes toward homosexual men. J Sex Res. 2013;50(3-4):329-52.

14. Costa DR, Bandeira HC, Nardi HC. Nardi Systematic review of instruments measuring homophobia and related constructs. J Appl Soc Psychol. 2013;43(6):1324-32.

15. Miller DB, Briggs H, Corcoran K. Fear of AIDS and Homophobia Scales: additional estimates of reliability and validity. Psychol Rep. 1997;81(3 Pt 1):783-6.

16. Campo-Arias A, Lafaurie MM, Gaitán-Duarte HG. Confiabilidad y validez de la escala para homofobia en estudiantes de medicina. Rev Colomb Psiquiatr. 2012;41(4):867-80.

17. Campo-Arias A, Oviedo-Acevedo HC, Herazo-Acevedo E. Desempeño psicométrico de la escala para homofobia en estudiantes de medicina. Rev Cinc Biomed. 2014;5:287-94.

18. Campo-Arias A, Oviedo H, Herazo E. Escala para homofobia: validez y confiabilidad en estudiante de medicina de una universidad de Bogotá (Colombia), 2010. Arch Med (Manizales). 2014;14:9-20.

19. Campo-Arias A, Herazo E, Oviedo HC. Escala breve para homofobia en estudiantes de Medicina de dos universidades colombianas: resultados de un proceso de refinamiento. Rev Colomb Psiquiat. 2017; 46:31-5.

20. Roberts $P$, Priest $H$, Traynor M. Reliability and validity in research. Nurs Stand. 2006;20(44):41-5.

21. Campo-Arias A, Herazo E. Homofobia en estudiantes de medicina: una revisión de los diez últimos años. Medunab. 2008;11:120-3.

22. Campo-Arias A, Díaz AJ, Herazo E. Homofobia en estudiantes de odontología e higiene oral: una revisión sistemática de la última década. Rev CES Odontol. 2008;21:63-8.

23. Meyer IH. Prejudice as stress: conceptual and measurement problems. Am J Public Health. 2003;93(2):262-5.

24. Hatzenbuehler ML, Phelan JC, Link BG. Stigma as a fundamental cause of population health inequalities. Am J Public Health. 2013;103(5):81321. 
25. Zambrano GE, Escalante HE. Grado de homofobia en estudiantes de enfermería de una universidad pública en Colombia. Rev Cienc Cuidado. 2013;10:115-26.

26. Campo-Arias A, Herazo E, Cogollo Z. Homofobia en estudiantes de enfermería. Rev Esc Enferm USP. 2010;44(3):839-43.

27. Sanchez NF, Rabatin J, Sanchez JP, Hubbard S, Kalet A. Medical students' ability to care for lesbian, gay, bisexual, and transgendered patients. Fam Med. 2006;38(1):21-7.

28. Bogotá. Ministerio de Salud. Resolución 008430 por la cual se establecen las normas científicas, técnicas y administrativas para la investigación en salud. Santa Fe de Bogotá: Ministerio de Salud; 1993.

29. Campo-Arias A, Oviedo HC. Propiedades psicométricas de una escala: la consistencia interna. Rev Salud Publica (Bogota). 2008 ;10(5):831-9.

30. Campo-Arias A, Herazo E, Oviedo HC. Análisis de factores: fundamentos para la evaluación de instrumentos de medición en salud mental. Rev Colomb Psiquiatr. 2012; 41(3):659-71.

31. Kaiser HF. An index of factorial simplicity. Psychometrika. 1974;34(1):31-6.

32. Bartlett MS. Test of significance in factor analysis. $\mathrm{Br} \mathrm{J}$ Psychol. 1950;3:77-85.

33. Cronbach J. Coefficient alpha and the internal structure of test. Psychometrika. 1951;16(3):297-334.

34. McDonald RP. Theoretical foundations of principal factor analysis and alpha factor analysis. Br J Math Stat Psychol. 1970;23:1-21.

35. Norman GR, Streiner DL. Bioestadística. Madrid: Mosby/Doyma Libros; 1996.

36. STATA 13.0. College Station: STATA; 2013.

37. Keszei AP, Novak M, Streiner DL. Introduction to health measurement scales. J Psychosom Res. 2010;68(4):319-23.
38. Macía F. Validez de los tests y análisis factorial: nociones generales. Cienc Trab. 2010;35:276-80.

39. Gorsuch RL. Exploratory factor analysis: its role in item analysis. J Pers Assess. 1997;68(3):532-60.

40. Reise SP, Waller NG, Comrey AL. Factor analysis and scale revision. Psychol Assess. 2000;12(3):287-97.

41. Skinner CJ, Henshaw PC, Petrak JA. Attitudes to lesbians and homosexual men: medical students care. Sex Transm Infect. $2001 ; 77(2): 147-8$.

42. Arnold 0, Voracek M, Musalek M, Springer-Kremser M. Austrian medical students' attitudes towards male and female homosexuality: a comparative survey. Wien Klin Wochenschr. 2004;116(21-22):730-6.

43. Alio AP, Makhale L, Hornschuh S, Hlongwane K, Otwombe K, Keefer M, et al. "Loving the sinner, hating the sin": an investigation of religious leaders' perceived role in the lives of persons living with HIV in Soweto, South Africa. J Glob Health Rep. 2019;3:e2019021.

44. Kan RW, Au KP, Chan WK, Cheung LW, Lam CY, Liu HH, et al. Homophobia in medical students of the University of Hong Kong. Sex Educ. 2009;9(1):65-80.

45. Hatzenbuehler ML, Phelan JC, Link BG. Stigma as a fundamental cause of population health inequalities. Am J Public Health. 2013;103(5):81321.

46. Pineda Roa CA. [Factors Associated with Suicide in Adolescents and Young People Self-Identified as Lesbian, Gay, and Bisexual: Current State of the Literature]. Rev Colomb Psiquiatr. 2013;42(4):333-49.

47. Lucassen MF, Stasiak K, Samra R, Frampton CM, Merry SN. Sexual minority youth and depressive symptoms or depressive disorder: A systematic review and meta-analysis of population-based studies. Aust N Z J Psychiatry. 2017 ;51(8):774-87. 